\title{
VALUE ADDITION TO CARTOSAT-I IMAGERY
}

\author{
Murali Mohan \\ Mobiterra Solutions (India) Private Limited, India \\ photogrammetry@ rediffmail.com; mohan@mobiterrasolutions.com
}

Commission VI, WG VI/4

KEY WORDS: RPCs, Block adjustment, stereo, imaging satellite, Cartosat-I, orthoimage, registration

\begin{abstract}
:
In the sector of remote sensing applications, the use of stereo data is on the steady rise. An attempt is hereby made to develop a software suite specifically for exploitation of Cartosat-I data. A few algorithms to enhance the quality of basic Cartosat-I products will be presented. The algorithms heavily exploit the Rational Function Coefficients (RPCs) that are associated with the image. The algorithms include improving the geometric positioning through Bundle Block Adjustment and producing refined RPCs; generating portable stereo views using raw / refined RPCs autonomously; orthorectification and mosaicing; registering a monoscopic image rapidly with a single seed point. The outputs of these modules (including the refined RPCs) are in standard formats for further exploitation in $3^{\text {rd }}$ party software. The design focus has been on minimizing the user-interaction and to customize heavily to suit the Indian context. The core libraries are in $\mathrm{C} / \mathrm{C}^{++}$and some of the applications come with user-friendly GUI. Further customization to suit a specific workflow is feasible as the requisite photogrammetric tools are in place and are continuously upgraded. The paper discusses the algorithms and the design considerations of developing the tools. The value-added products so produced using these tools will also be presented.
\end{abstract}

\section{INTRODUCTION}

\subsection{The satellite}

Cartosat-I, of 2005 vintage, continues to beam stereo pairs from its twin cameras at a ground pixel resolution better than $2.5 \mathrm{~m}$ : one component near-nadir-looking and the other obliquelooking. A stereo pair sweeps ground area of 800 sq. km. nearly. The two stereo components, intuitively labelled AFT and FORE images to indicate the look direction, are designed to produce Digital Elevation Models (DEMs) highly amenable to autocorrelation - possible due to near-simultaneous imaging. The data from on-board instruments like GPS and improvised star sensor are used to generate terrain-independent Rational Function Model.

\subsection{RPC characteristics}

Rational Function Coefficients (RPCs), bundled with GeoTiff images by the data provider, enable two-way relation between the image and object spaces, obviating the knowledge of the imaging system. Such vendor-side RPCs are sensor-derived and terrain-independent. A sparse but nation-wide control point library aids in producing quality RPCs which produce parallaxfree stereo models. However, they require refinement at scene/block level for attaining mapping accuracy by introducing GCPs by the end-user (Sadasiva Rao etal, 2006) The model adapted in this exercise is categorized as "terrain-dependent model scenario; user-side RPC refinement". For comprehensive treatment on this topic (Tao and $\mathrm{Hu}, 2001$ ) may be referred. The salient behaviour of RPCs is discussed by Fraser and Hanley (2003).

The photogrammetric systems do carry out the required refinement through Block Adjustment and then generally encapsulate the refined RPCs in proprietary formats, thus preventing their direct interoperability across the systems.

The observation equations are of the following form:

$$
\begin{aligned}
& \text { row }=\frac{\left(\begin{array}{lllllllllll}
1 & Z & Y & X & \ldots & Y^{3} & X^{3}
\end{array}\right) \cdot\left(\begin{array}{lllllll}
a_{0} & a_{1} & \ldots & a_{19}
\end{array}\right)^{T}}{\left(\begin{array}{llllllllll}
1 & Z & Y & X & \ldots & Y^{3} & X^{3}
\end{array}\right) \cdot\left(\begin{array}{llllll}
1 & b_{1} & \ldots & b_{19}
\end{array}\right)^{T}} \\
& \text { column }=\frac{\left(\begin{array}{llllllllll}
1 & Z & Y & X & \ldots & Y^{3} & X^{3}
\end{array}\right) \cdot\left(\begin{array}{ccccccc}
c_{0} & c_{1} & \ldots & c_{19}
\end{array}\right)^{T}}{\left(\begin{array}{llllllllll}
1 & Z & Y & X & \ldots & Y^{3} & X^{3}
\end{array}\right) \cdot\left(\begin{array}{llllll}
1 & d_{1} & \ldots & \left.d_{19}\right)^{T}
\end{array}\right.}
\end{aligned}
$$

where (row, column) $=$ the image-space coordinates $(\mathrm{X}, \mathrm{Y}, \mathrm{Z})=$ the ground-space coordinates $(\mathrm{a}, \mathrm{b}, \mathrm{c}) \mathrm{s}=$ the coefficients (RPCs)

Further mathematical treatment can be found in Grodecki and Dial,(2001) and Fraser etal (2006). The subtle distinction between the types of RPCs is described in the following subsections.

\subsubsection{Vendor-side RPCs}

The vendor of the imagery (e.g., ISRO for Cartosat-I) has access to the physical parameters of the sensor. The RPCs so generated using sparse GCPs are largely terrain-independent. Rational Function Model, provided in the form of Orthokit by NRSC Data Centre (NDC) is considered here as the base input amenable for value-addition.

\subsubsection{User-side RPCs}

When the end-user procures imagery along with RPCs (produced by the vendor as described above), it is required to further refine the RPCs with the GCPs through the photogrammetric process of Block Adjustment. Using the refined RPCs, the forward processing for stereo-compilation, DEM generation and ortho-rectification can be taken up. The 
appendix lists a sample of vendor-side and user-side RPCs for the same image.

\section{DEVELOPMENT OF PHOTOGRAMMETRIC LIBRARY}

\subsection{Design philosophy}

The endeavour is to design and develop a set of photogrammetric tools exclusively for satellite stereo processing. Where possible inbuilt linkages are provided through organised reference data (such as gratis DEMs, map grids). RPCs are extensively used as the principal sensor model - considering its popular use and generality. Where RPC formats are differently packaged from Ikonos / Cartosat format, suitable parsers are developed (e.g., Pleiades, WorldView).

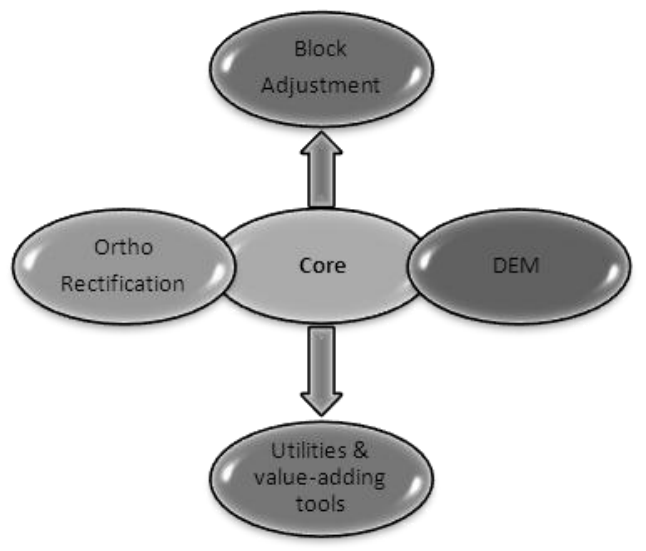

Figure 1. The scope of the library design

The following stand-alone modules have so far been developed and tested for the targeted usage:

\begin{tabular}{|l|l|}
\hline Name of the module & \multicolumn{1}{c|}{ Brief functionality } \\
\hline TerraBlock & $\begin{array}{l}\text { Block adjustment of stereo pairs and } \\
\text { producing open-formatted refined } \\
\text { user-side RPCs } \\
\text { Ortho-rectification and mosaicing }\end{array}$ \\
TiffTiler & $\begin{array}{l}\text { Slicing based on SoI map index } \\
\text { Anaglyph generation using raw or } \\
\text { refined RPCs }\end{array}$ \\
Portable Stereo Tool & $\begin{array}{l}\text { Image registration with one seed } \\
\text { point }\end{array}$ \\
\hline
\end{tabular}

Table 1. Developed and tested modules

\subsection{TerraBlock: Block Adjustment}

The scope of the module is to allow the user to mark the control and tie points and then perform the bundle block adjustment. The output is a report on adjustment and the refined RPCs in plain text format. The input images and the corresponding raw RPCs are organised in a folder. The program checks the adequacy and correspondence of RPCs first. It generates the thumbnail images of all the input images on an informative window. The control points are input in ESRI Shape file format Upon selecting a point for marking, the software guesses the images likely to contain the point and focuses to the approximate location within the image, aiding the user to complete the task of measurements faster. The principal functions of the module are tabulated below:

\begin{tabular}{|c|c|}
\hline $\begin{array}{l}\text { Ground to Image } \\
\text { Function [To find } \\
\text { image coordinates for } \\
\text { the given ground } \\
\text { coordinates.] }\end{array}$ & $\begin{array}{l}\text { RFM } \rightarrow \text { Apply_G2I( long, lat, } \\
\text { height, scan, pixel ); } \\
\text { or, } \\
\text { RFM } \rightarrow \text { Apply_G2I(ground co- } \\
\text { ordinate file, image co-ordinate } \\
\text { file); }\end{array}$ \\
\hline $\begin{array}{l}\text { Image to Ground } \\
\text { Function [For given } \\
\text { image coordinates } \\
\text { and height, it finds } \\
\text { the corresponding } \\
\text { ground coordinates. } \\
\text { ] }\end{array}$ & $\begin{array}{l}\text { RFM } \rightarrow \text { Apply_I2G( scan, pixel, } \\
\text { height, long, lat ); } \\
\text { Or, } \\
\text { RFM } \rightarrow \text { Apply_G2I(image co- } \\
\text { ordinate file, ground co-ordinate } \\
\text { file); }\end{array}$ \\
\hline $\begin{array}{l}\text { Stereo Intersection } \\
\text { [Finds the 3D ground } \\
\text { co-ordinates for } 2 \text { or } \\
\text { more images covering } \\
\text { the object.] }\end{array}$ & $\begin{array}{l}\text { RFM } \rightarrow \text { Intersection (); } \\
\text { RFM } \rightarrow \text { Core_Intersection(scanline, } \\
\text { pixel, long, lat, height); } \\
\text { RFM } \rightarrow \text { Free_Intersection(); } \\
\quad \text { Or, } \\
\text { RFM } \rightarrow \text { Intersection( tie-points file, } \\
\text { lat-long-height file); }\end{array}$ \\
\hline $\begin{array}{l}\text { Bundle Adjustment } \\
\text { [Estimates the } \\
\text { corrections for each } \\
\text { of the block images, } \\
\text { does the block } \\
\text { adjustment, and } \\
\text { computes the ground } \\
\text { coordinates of the tie } \\
\text { points.] }\end{array}$ & $\begin{array}{l}\text { RFM } \rightarrow \text { doBundleAdjust (variable); } \\
\begin{array}{r}\text { Variable }=0 \text { for Bias corrections } \\
\quad=1 \text { for Affine corrections }\end{array}\end{array}$ \\
\hline $\begin{array}{l}\text { Fitting the RPCs } \\
\text { [RPC fit-errors on } \\
\text { check points] }\end{array}$ & $\begin{array}{l}\text { RPC_Process *RFM; RFM=new } \\
\text { RPC_Process; } \\
\text { RFM } \rightarrow \text { Fit_RPC }() ; \\
\text { RFM } \rightarrow \text { Check_RPC }() ; \text { Delete } \\
\text { RFM; }\end{array}$ \\
\hline
\end{tabular}

Table 2. Principal components of the photogrammetric core library

The module has been tested with the stereo data of Cartosat-I and GeoEye (Murali Mohan, 2011). Add-on functionalities for handling WorldView stereo and Pleiades triplet are being developed.

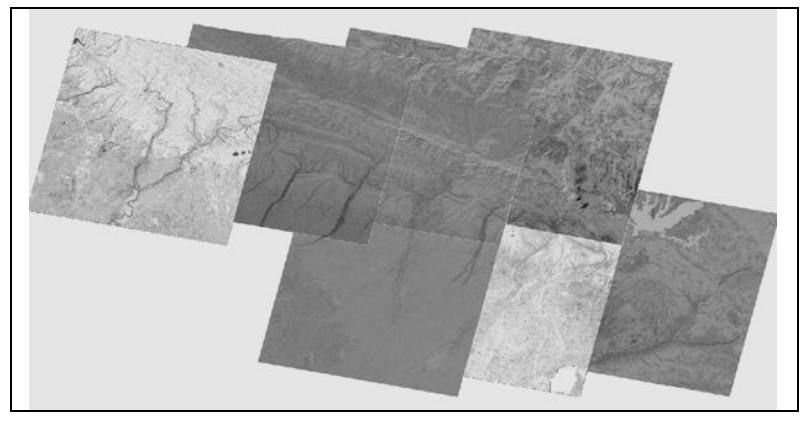

Figure 2. The stereo pairs of Cartosat-I used for testing the accuracy

The module computes the refined RPCs and writes them as text files - enabling their exploitation in other photogrammetric software for the tasks like DEM extraction 
and feature coding. The appendix lists the raw and refined RPCs to demonstrate this compatibility. The module comes with ease-of-use GUI that enables manual marking of control and tie points. Control requirement is one common point for four stereo models.

In the use-case of 7 stereo models of Cartosat, wherein 9 GCPs are used and the accuracy is checked at 132 check points. The errors at control points are $0.8,0.5$ and $1.3 \mathrm{~m}$ in $\mathrm{XYZ}$ respectively; and at the check points the RMS in XY is $2.6 \mathrm{~m}$ and $\mathrm{RMS}$ in $\mathrm{Z}$ is $3.1 \mathrm{~m}$. These are in-tune with the benchmarking findings (Sadasiva Rao etal, 2006).

\subsection{RectiFire: Orthorectification and mosaicing}

A logical follow-up operation after refining the RPCs is to ortho-rectify the image(s) so desired. RectiFire is a standalone module which processes individual scenes and a seamless mosaic is generated with a preview option. The module extracts the corresponding DEM from the linked data repository (containing SRTM and ASTER) for the extent of the scene automatically, thus minimizing the processing steps. The sample output of the module is given below:

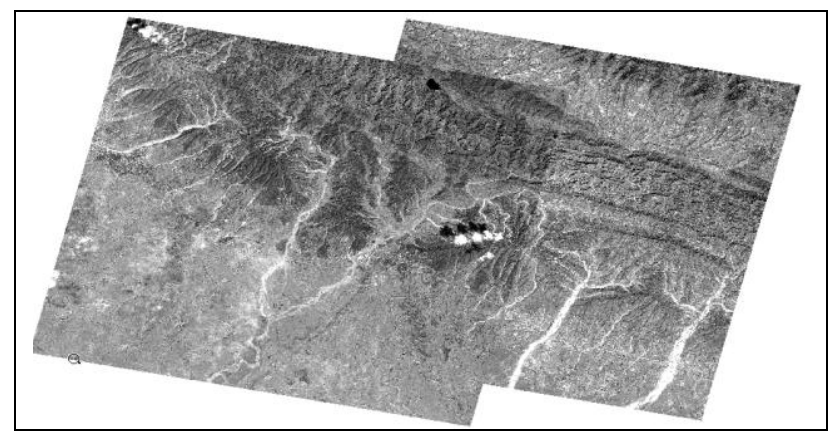

Figure 3. The mosaic of ortho images resulting from adjacent paths of the satellite

In addition, provisions for constant elevation based correction for flat areas and user-provided DEM are built-in. The most commonly used resampling options are incorporated. The output bit-depth can be $8 / 16$ bits. The geographic framework is WGS84.

\subsection{TiffTiler: Ortho-slicing based on national map grid}

Further, user can slice the orthoimages based on Survey of India (SoI) map index at 1: 25000 scale. The index-grid is built-in and hence the operation avoids user-inputs. TiffTiler identifies the partial orthos resulting from overlapping orthos and mosaics them automatically where possible. The module handles 8/16/24-bit GeoTiff. A cut-off threshold of minimum $10 \%$ image data per map sheet eliminates sliver tiles.

\subsection{Portable Stereo Tool: Anaglyph generation}

This is an innovative tool towards value-addition. Making use of the raw or refined RPCs, the tool carries out the Epipolar registration of the stereo-pair and generates the anaglyph on-thefly. The output is printable after the map-composition. The process does not require DEM or DTM - thus obviating the limitation of the subtle terrain-defining details such as troughs and tops being missed out due to coarse cell-spacing.
The tool handles the NRSC-supported stereo pairs or customgenerated long strips. Multiple options for choosing the area of interest are incorporated such as SoI map sheet extents, pointradius, bounding box, or complete model.

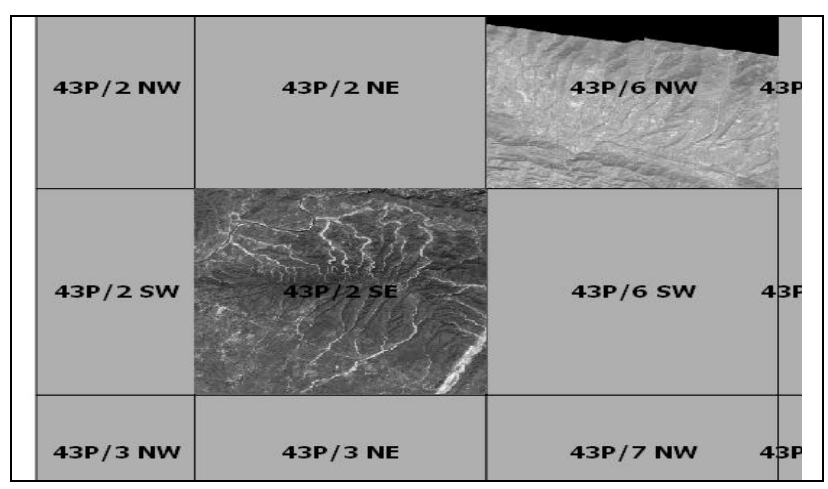

Figure 4. The orthoimage being sliced as per the Survey of India map extent definition/

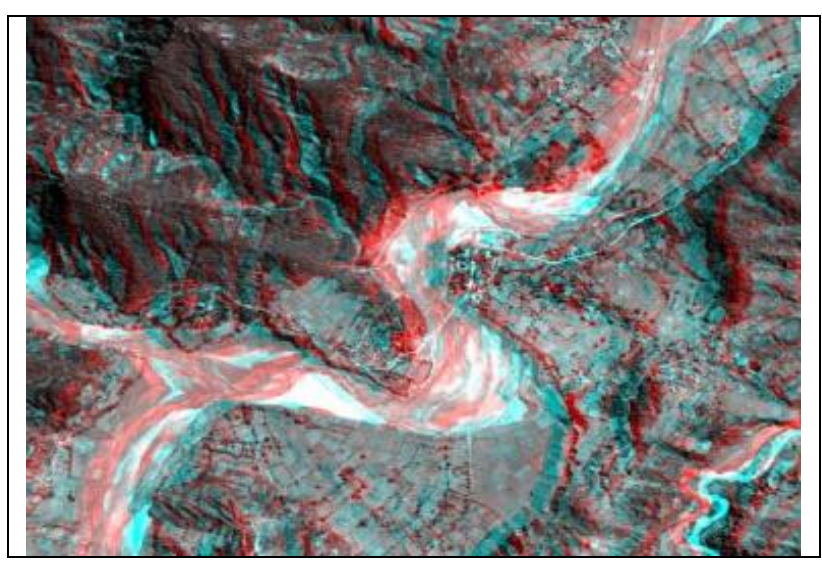

Figure 5. The anaglyph generated from a stereo pairs of Cartosat-I with raw RPCs. (To appreciate the depth, the RedCyan filters are to be used as eye-wear.)

The anaglyphs can be inserted into project reports for enhanced terrain appreciation. When carried to the field, the output aids in authentic correlation with the terrain the user is standing on.

\subsection{EasyReg: Image Registration made easy}

This is a standalone tool to quickly register Cartosat I and II satellite imagery by exploiting the associated raw RPCs. The salient feature is the requirement of minimal user-interaction limited to adding one seed point. The tool comes embedded with the reference datasets-to collect the seed point; and later to do the quality check. The built-in functions such as horizontal and vertical swiping aid the checking process. The whole exercise of registration is designed in such a way that it seldom exceeds 10 minutes of elapsed time (excluding the raw image ingest time). This approach is preferred when the user is required to geometrically rectify a single image without going through the conventional photogrammetric process.

\section{CONCLUSIONS}

The set of photogrammetric tools for value-addition to satellite stereo imagery has been presented. The design aspects and sample results of block adjustment, ortho-rectification, portable stereo generation and image registration are discussed. The 
outputs of these modules are interoperable with commercial photogrammetric systems and open GIS packages. The modules have been tested successfully and the results are commensurate with the mission capabilities of Cartosat-I.

The refined RPCs written in open format offers great flexibility to perform tasks in preferred systems by the end-user. They enable DEM and stereo-compilation directly.

\section{REFERENCES}

Cartosat-1 (IRS-P5) Data Products Generation Station: DDR Document. Signal and Image Processing Group, Space Applications Centre, Ahmedabad, April 2004

Fraser, C., Dial, G., Grodecki, J., 2006. Sensor orientation via RPCs. ISPRS Journal of Photogrammetry \& Remote Sensing 60(3): 182-194.

Fraser, C. S., H. B. Hanley, 2003. Bias compensation in rational functions for Ikonos satellite imagery.

Photogrammetric Engineering and Remote Sensing, 69(1): pp. 53-57.

Grodecki, J., and Dial, G., 2001, IKONOS Geometric Accuracy, Proceedings of Joint ISPRS Workshop on High Resolution Mapping from space, 19-21 September, pp. 77-86

Murali Mohan ASRKV., Block Adjustment of Cartosat-I Stereo Data Using RPCs, Geospatial World Forum, 2011.

Sadasiva Rao, B., Murali Mohan ASRKV., Kalyanaraman, K., Radhakrishnan, K. Evaluation of Cartosat-I Stereo Data of Rome, 2006, International Archives of Photogrammetry, Remote Sensing and Spatial Information Sciences, Vol. 36, Part 4, on CD-ROM. Also in Vol. XXXVI, Part-IVB, pp. 10261029 .

Tao, C. V., Y. Hu , 2001. A comprehensive study on the rational function model for photogrammetric processing. Photogrammetric Engineering and Remote Sensing, 67(12): pp. 1347-1357.

\section{APPENDIX}

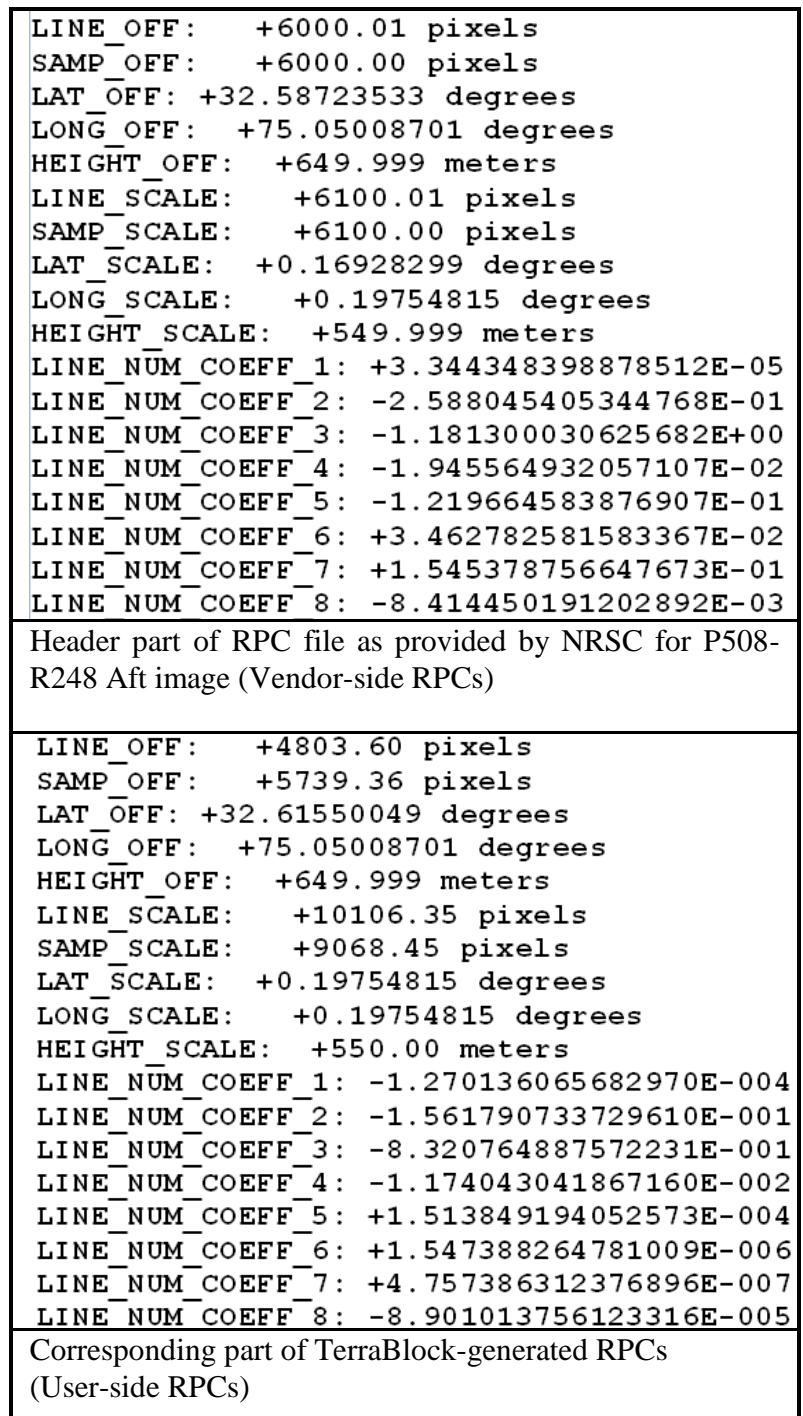

